Western University

Scholarship@Western

Medical Biophysics Publications

Medical Biophysics Department

$2-2015$

Feasibility of a unified approach to intensitymodulated radiation therapy and volumemodulated arc therapy optimization and delivery

Jerry J. Battista

University of Western Ontario, j2b@uwo.ca

Michael MacFarlane

Western University, mmacfar4@uwo.ca

Eugene Wong

University of Western Ontario, ewong4@uwo.ca

JeffZ. Chen

Western University, jchen4@uwo.ca

Douglas A. Hoover

Western University, dhoover3@uwo.ca

Follow this and additional works at: https://ir.lib.uwo.ca/biophysicspub

Part of the Medical Biophysics Commons

Citation of this paper:

Battista, Jerry J.; MacFarlane, Michael; Wong, Eugene; Chen, Jeff Z.; and Hoover, Douglas A., "Feasibility of a unified approach to intensity-modulated radiation therapy and volume-modulated arc therapy optimization and delivery" (2015). Medical Biophysics Publications. 15.

https://ir.lib.uwo.ca/biophysicspub/15 


\section{Feasibility of a unified approach to intensity-modulated radiation therapy and volume-modulated arc therapy optimization and delivery}

Douglas A. Hoover, Michael MacFarlane, Eugene Wong, Jerry J. Battista, and Jeff Z. Chen

Citation: Medical Physics 42, 726 (2015); doi: 10.1118/1.4905373

View online: http://dx.doi.org/10.1118/1.4905373

View Table of Contents: http://scitation.aip.org/content/aapm/journal/medphys/42/2?ver=pdfcov

Published by the American Association of Physicists in Medicine

\section{Articles you may be interested in}

Sci-Fri AM: Mountain - 05: Unified Optimization and Delivery of Intensity-modulated Radiation Therapy and Volume-modulated Arc Therapy

Med. Phys. 41, 24 (2014); 10.1118/1.4894945

Planning and delivery of intensity-modulated radiation therapy

Med. Phys. 35, 5233 (2008); 10.1118/1.3002305

A toolbox for intensity modulated radiation therapy optimization

Med. Phys. 30, 2320 (2003); 10.1118/1.1598171

MAGIC-type polymer gel for three-dimensional dosimetry: Intensity-modulated radiation therapy verification Med. Phys. 30, 1264 (2003); 10.1118/1.1576392

Dose to radiation therapists from activation at high-energy accelerators used for conventional and intensitymodulated radiation therapy

Med. Phys. 29, 598 (2002); 10.1118/1.1463063

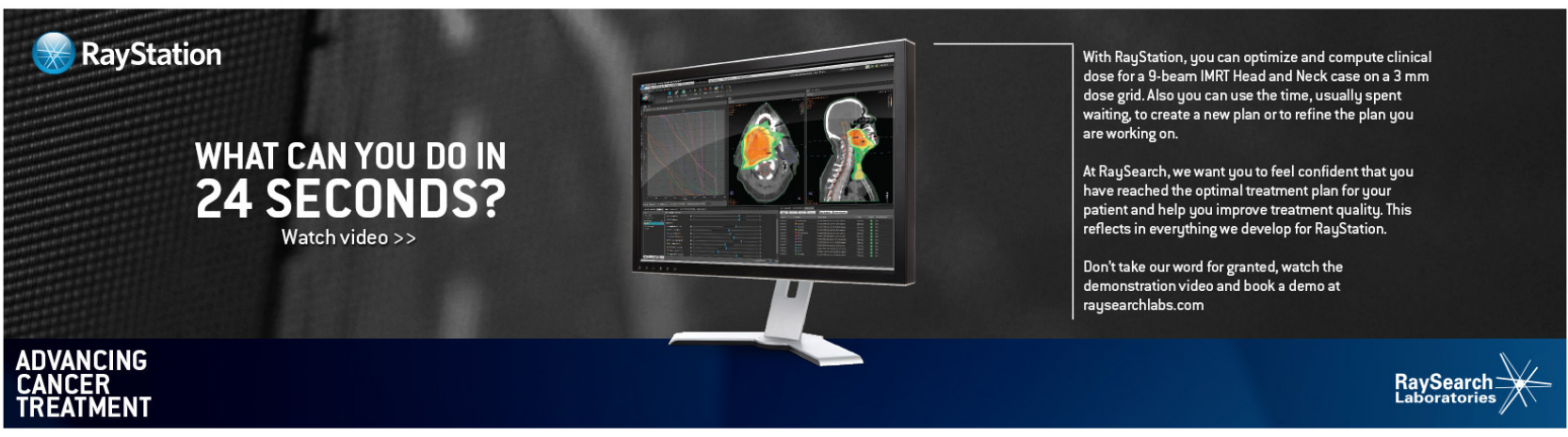




\title{
Feasibility of a unified approach to intensity-modulated radiation therapy and volume-modulated arc therapy optimization and delivery
}

\author{
Douglas A. Hoovera) \\ Department of Physics and Engineering, London Regional Cancer Program, London, Ontario N6A 4L6, \\ Canada; Department of Oncology, Western University, London, Ontario N6A 3K7, Canada; \\ and Department of Medical Biophysics, Western University, London, Ontario N6A 3K7, Canada \\ Michael MacFarlane \\ Department of Physics and Engineering, London Regional Cancer Program, London, Ontario N6A 4L6, \\ Canada and Department of Medical Biophysics, Western University, London, Ontario N6A 3K7, Canada
}

Eugene Wong

Department of Oncology, Western University, London, Ontario N6A 3K7, Canada; Department of Medical Biophysics, Western University, London, Ontario N6A 3K7, Canada; and Department of Physics and Astronomy, Western University, London, Ontario N6A 3K7, Canada

Jerry J. Battista

Department of Physics and Engineering, London Regional Cancer Program, London, Ontario N6A 4L6, Canada; Department of Oncology, Western University, London, Ontario N6A 3K7, Canada; Department of Medical Biophysics, Western University, London, Ontario N6A 3K7, Canada; and Department of Physics and Astronomy, Western University, London, Ontario N6A 3K7, Canada

\section{Jeff Z. Chen}

Department of Physics and Engineering, London Regional Cancer Program, London, Ontario N6A 4L6, Canada; Department of Oncology, Western University, London, Ontario N6A 3K7, Canada; and Department of Medical Biophysics, Western University, London, Ontario N6A 3K7, Canada

(Received 11 July 2014; revised 17 December 2014; accepted for publication 19 December 2014; published 14 January 2015)

Purpose: To study the feasibility of unified intensity-modulated arc therapy (UIMAT) which combines intensity-modulated radiotherapy (IMRT) and volumetric-modulated arc therapy (VMAT) optimization and delivery to produce superior radiation treatment plans, both in terms of dose distribution and efficiency of beam delivery when compared with either VMAT or IMRT alone.

Methods: An inverse planning algorithm for UIMAT was prototyped within the PINNACLE treatment planning system (Philips Healthcare). The IMRT and VMAT deliveries are unified within the same arc, with IMRT being delivered at specific gantry angles within the arc. Optimized gantry angles for the IMRT and VMAT phases are assigned automatically by the inverse optimization algorithm. Optimization of the IMRT and VMAT phases is done simultaneously using a direct aperture optimization algorithm. Five treatment plans each for prostate, head and neck, and lung were generated using a unified optimization technique and compared with clinical IMRT or VMAT plans. Delivery verification was performed with an ArcCheck phantom (Sun Nuclear) on a Varian TrueBeam linear accelerator (Varian Medical Systems).

Results: In this prototype implementation, the UIMAT plans offered the same target dose coverage while reducing mean doses to organs at risk by $8.4 \%$ for head-and-neck cases, $5.7 \%$ for lung cases, and $3.5 \%$ for prostate cases, compared with the VMAT or IMRT plans. In addition, UIMAT can be delivered with similar efficiency as VMAT.

Conclusions: In this proof-of-concept work, a novel radiation therapy optimization and delivery technique that interlaces VMAT or IMRT delivery within the same arc has been demonstrated. Initial results show that unified VMAT/IMRT has the potential to be superior to either standard IMRT or VMAT. (c) 2015 American Association of Physicists in Medicine. [http://dx.doi.org/10.1118/1.4905373]

Key words: IMRT, VMAT, treatment planning, optimization

\section{INTRODUCTION}

Volumetric-modulated arc therapy (VMAT) has been rapidly adopted by the radiotherapy community due primarily to its delivery speed and monitor unit (MU) efficiency, as well as the quality of conformal dose distributions achievable. ${ }^{1-3}$
On the other hand, intensity-modulated radiotherapy (IMRT) with its static beam directions might be advantageous in cases where steep dose gradients or highly intensity-modulated beam intensities are required in preferred directions. ${ }^{4}$ While the community tends to regard these two delivery techniques as disparate entities, they are in reality special cases of one 
another. More specifically, there exists a unifying delivery technique which bridges the gap between static-gantry IMRT and rotating-gantry VMAT. Such a unified delivery, if properly implemented into an inverse-planning algorithm, would in general lead to improved dose delivery capabilities as the algorithm could naturally optimize the beam within a given arc range to be more IMRT-like if greater beam intensity modulation is required, or more VMAT-like if increased conformity is required with less beam intensity modulation. This combined approach would take advantage of the two modes of beam delivery for targeting and normal tissue sparing.

IMRT or VMAT delivery is typically represented by control points (CP)—or multileaf collimator (MLC) segmentswhich are essentially snapshots of the positions of the relevant linear accelerator components taken at regular intervals. For example, a VMAT beam can be stored as a series of control points specifying the machine parameters at regular $2^{\circ}$ or $4^{\circ}$ intervals of the rotating gantry. Due to the large number of degrees-of-freedom required to specify a VMAT beam, all VMAT radiotherapy plans are created using specialized optimization software, such as SmartArc in PINNACLE (Philips Healthcare, Fitchburg) or RapidArc in Eclipse (Varian Medical Systems, Palo Alto). Both commercial treatment planning systems optimize beam parameters specifically for equally spaced gantry positions. In addition to commercially available software, a number of research prototypes have been developed to test novel optimization algorithms including the concept of unequally spaced control points. ${ }^{5,6}$ In principle, VMAT and IMRT with and without gantry rotation, respectively, can be unified during optimization and delivery. Instead of forcing the beam angle increments for arc therapy, the fixed-gantry IMRT parameters can add additional degrees of freedom available to the optimization engine.

The idea to combine VMAT and IMRT deliveries is not new, and various ideas have been proposed to improve the current clinically available VMAT implementations. One suggestion, termed dense angularly sampled and sparse intensity modulated radiation therapy (DASSIM-RT), proposes a method to search more thoroughly through the parameter space of machine-deliverable radiotherapy plans. $^{7}$ One major hurdle toward the clinical implementation of this method is that the scale of the optimization problem is now immense and so much greater computer memory is required. ${ }^{8}$ Another proposal, arc modulated radiation therapy (AMRT), builds on previous work in intensity modulated arc therapy (IMAT). ${ }^{1}$ AMRT is a sequencing algorithm that allows multiple IMAT arcs to be delivered by a single arc. In one study, AMRT plans required on the order of 200-400 segments and achieved similar plan quality to IMRT. ${ }^{9}$

Yet another proposal, termed FusionArc, has recently been published. ${ }^{10}$ This paper describes an in-house optimization software that begins with a full VMAT optimization followed by an iterative conversion, at select gantry angles, from uniform to intensity-modulated fluence. Gantry angles are selected for conversion based on a gradient function, which attempts to predict conversions that will lead to the greatest reduction in the cost function. Brainlab (Feldkirchen,
Germany) has introduced a commercial platform, which first optimizes a dynamic conformal arc delivery phase followed by an optimization of an IMRT phase. However, the arc phase is restricted by constant angular speed and dose rate. Furthermore, the arc ranges, the number of uniformly spaced IMRT fields, and the relative weighting of arc and fixed-gantry IMRT phases must all be chosen from the outset of optimization. ${ }^{11}$ The quality of HybridArc plans has been shown to depend on a proper selection of these initial parameters. ${ }^{12}$

None of the solutions described above has yet embodied the fully integrated and unified approach that we advocate here. The purpose of this work is to create, as a proof-ofprinciple, a unified intensity-modulated arc therapy (UIMAT) that combines IMRT and VMAT optimization and delivery in order to produce efficient and superior radiation treatment plans in a single optimization with concurrent VMAT and IMRT features.

\section{MATERIALS AND METHODS}

\section{A. Inverse planning for UIMAT}

Custom software was developed and integrated into a commercial treatment planning system, PINNACLE (Ref. 3) v9.6 Radiation Therapy Planning Systems (Philips Healthcare, Fitchburg) for the purpose of testing whether UIMAT is superior to either standard VMAT or IMRT. This software extends PINNACLE's built-in VMAT optimization in a few key ways. First, it removes the restriction that control points within a dynamic arc must have a uniform angular spacing. Importantly, this allows arc segments that would benefit from increased intensity modulation to have more densely packed control points. Furthermore, angles requiring this increased modulation are selected automatically during the initial optimization stage, and this can be thought of as a form of beam-angle optimization.

Our optimization algorithm begins by creating static beams uniformly distributed between start and stop arc angles with an initially coarse control point spacing of $24^{\circ}$, as suggested by Bzdusek. ${ }^{13}$ Using PINNACLE's inverse-planning system, an optimized fluence pattern is determined for these initial beams. In general, these fluence patterns will not be machinedeliverable and so a sequencing step is then performed, which converts the fluence patterns into executable MLC control points.

Custom software is then initiated which reads in this beam information from PINNACLE software. As a preparatory step, the code first reorders the control points within each individual beam, anticipating that these control points may later be distributed —or fanned out—into other gantry angles to form an arc segment. The goals of this reordering step are (1) to preferentially place higher-weighted control points toward the middle of the order, thereby minimizing the dosimetric differences between the original and the fanned out beams (see Fig. 1); (2) to minimize MLC motion between control points, thereby improving delivery efficiency. Note that for any given solution, there will always be a "symmetric" solution having the reverse control point order. However, this symmetry is 


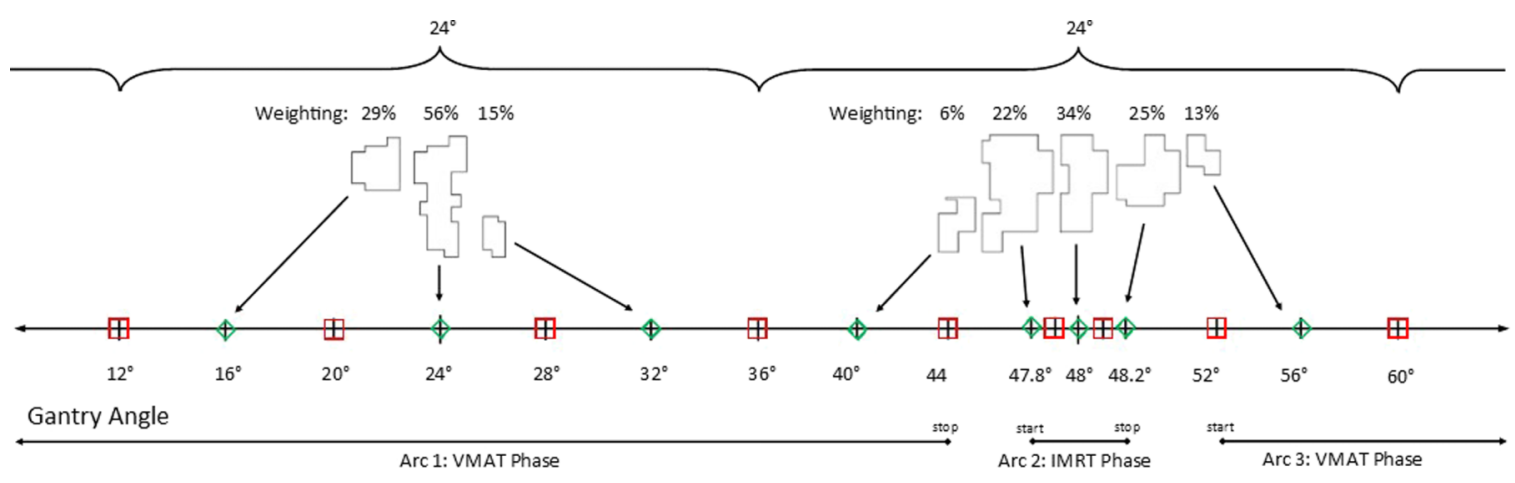

$\diamond=$ Original MLC Segments

$\square=$ Lin. Interp. MLC Segments

FIG. 1. Schematic showing how the control points from the initial fixed-beam optimization are distributed into VMAT and IMRT phases. Control points within each beam have been reordered to minimize MLC motion. As well, higher-weighted control points are preferentially placed in the middle of the ordering to minimize the difference between the initial and final gantry angles. Interpolated MLC segments are inserted as necessary to maintain a maximum control point spacing of four degrees.

broken once neighboring beams are considered, and so for each beam the software determines whether the original or reverse order will result in the least MLC motion between the first and last control points of neighboring beams.

The algorithm next determines which of these beams should remain as IMRT fields and which should be converted into arc segments. In our current implementation, we chose to convert those beams having fewer than four control points (low modulation) into a "VMAT phase," while those beams with four or more control points were retained for the "IMRT phase" (high modulation). In this study, the threshold to distinguish between low and high modulation corresponds approximately to the average number of control points per beam, which in turn depends on the MLC sequencing parameters chosen. Based on the parameters we chose, on average, we had four control points per beam, which we found to work well practically for the anatomical sites tested.

Finally, the algorithm modifies the gantry angle associated with each beam segment as shown in Fig. 1. Beams that have low modulation become VMAT phases where their control points are distributed uniformly within the $24^{\circ}$ spacing. In order to maintain approximately four-degree spacing between control points, linear interpolation of MLC leaves is used to create new interlaced control points as needed. Beams that have high modulation become IMRT phases. In this case, the first and last control points have their gantry angles shifted $8^{\circ}$ on either side of the initial gantry angle, as shown in Fig. 1. These two boundary control points, together with linearly interpolated control points, are used to facilitate a smooth transition from VMAT to IMRT phases. Next, the central control points of high-modulation beams are fanned out around the initial gantry angle in $0.2^{\circ}$ increments. Interpolated control points are then added between these central control points to arrive at a $0.1^{\circ}$ spacing for the IMRT phase. The small gantry rotation (almost stalled) for the IMRT phase approximates a static-gantry IMRT field and is a work-around for the fact that PINNACLE v9.6 does not allow simultaneous DMPO optimization of static-gantry IMRT and VMAT beams. At this stage, the beam control points are reimported into
PINNACLE for a final optimization using the DMPO algorithm. As an example, the start and stop angles for these partial arcs, as well as the angular location of the IMRT phases, are shown in Fig. 2.

Certain "soft" deliverability constraints are relaxed for the IMRT-like portions of delivery. Specifically, for the IMRT phases, the maximum MU per degree is increased from 20 to 200 , and the gantry acceleration limit is removed. Within PINNACLE software, this is accomplished by defining a separate machine having these special properties and associating the IMRT phases with this virtual machine. This is required in order to have a reasonable number of MUs delivered during the more highly modulated IMRT phase, which may have up to ten control points within one degree angular spacing. It is important to note that such a beam is still machine

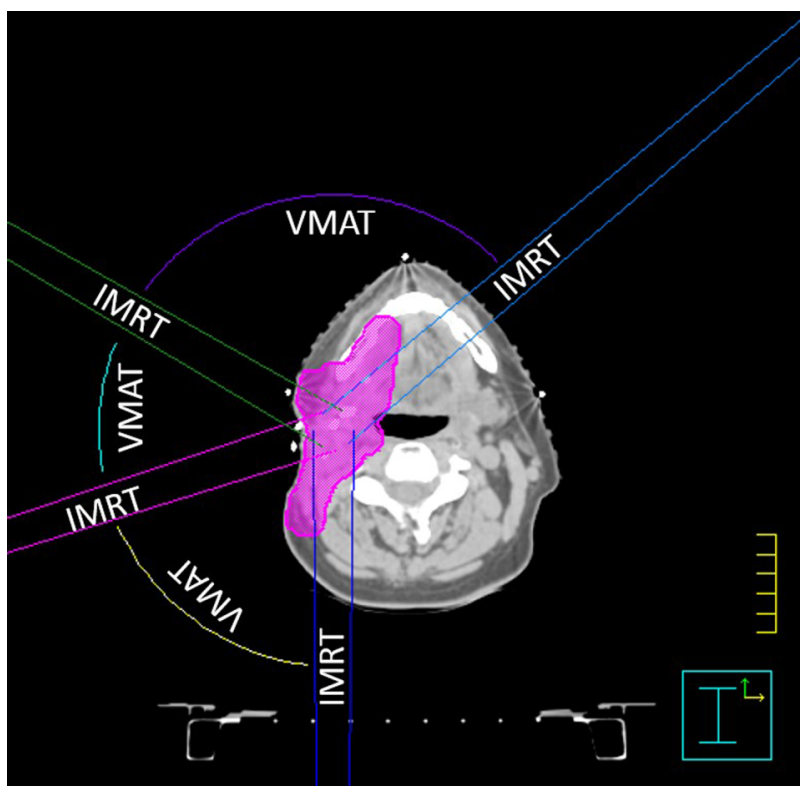

FIG. 2. A typical UIMAT plan. The angular ranges for three VMAT phases are represented by arc segments while the fixed gantry angles corresponding to four IMRT phases are represented by straight line pairs. 
deliverable as it does not violate any physical constraints of the accelerator.

From this point on, optimization proceeds using the standard functionality within PINNACLE. Machine parameters for both IMRT phases and VMAT phases are optimized at the same time as multiple dynamic arcs in PINNACLE software. DMPO optimization is continued until a clinically acceptable plan is obtained using standard dose-volume histogram (DVH) constraints. During optimization, the VMAT and IMRT phases are treated as separate beams, and after optimization is complete, our custom software is used to combine the VMAT and IMRT phases into a single UIMAT arc with variable gantry speed for final dose calculation and delivery. The final dose is calculated by collapsed cone convolution algorithm in the PINNACLE treatment planning system.

\section{B. Treatment plan evaluation}

In order to test our optimization algorithm, CT scans from 15 previously treated patients were selected for planning comparison purposes. Five cases each were randomly selected from head-and-neck, lung, and prostate sites to represent a variety of anatomy, complexity levels, and delivery modalities. All clinical treatment plans were optimized using the PINNACLE treatment planning system. A summary of the selected patient plans is provided in Table I with the estimated delivery times obtained from PINNACLE. Since PINNACLE does not provide an estimate for step-and-shoot beams, the delivery time for IMRT plans was measured on a Varian linear accelerator. The IMRT delivery time is defined here as the total time from when the first beam turns on until the last beam turns off and so includes the "mode-up" time as well as the time required to move to the various gantry positions.
Beams were ordered for the most efficient delivery. A paired, two-tailed $t$-test was used to assess for statistically significant differences in delivery times.

The UIMAT plans were optimized based on our local treatment planning guidelines used to generate the clinical plans. Without exception, critical structure tolerances such as the spinal cord and brainstem were respected. For lung cases, the volume of both lungs receiving at least $20 \mathrm{~Gy}(V 20)$ was kept below 35\% and a mean lung dose of $<20$ Gy was also maintained. The dose coverage goal for the planning target volume (PTV) was to cover at least $95 \%$ of the PTV by at least 95\% of the prescription dose. For noncritical structures, DVH criteria from RTOG 0126 and QUANTEC (Ref. 14) were used to guide the planning process. All UIMAT plans were restricted to a single arc. Dynamic jaw movements were allowed in order to facilitate the goal of a single-arc delivery. Dynamic jaws were required when treating large volumes with a single arc due to the finite speed and length of the MLC leaves. It is noted that the clinical plans did not require dynamic jaws, as all complicated VMAT plans standardly use two arcs.

To compare a UIMAT plan with the corresponding clinical VMAT or IMRT plan for each patient, various dose metrics were selected and subjected to a paired, one-tailed $t$-test to assess statistically significant differences. Mean doses for PTVs and conformity index (CI) were analyzed for all cases. The conformity index has been previously defined as ${ }^{15}$

$$
\begin{aligned}
\mathrm{CI} & =\text { cover factor } \times \text { spill factor } \\
& =\left(\frac{V 100(\mathrm{PTV})}{V_{\mathrm{PTV}}}\right) \times\left(\frac{V 100(\mathrm{PTV})}{V 100(\text { body })}\right),
\end{aligned}
$$

where $V 100$ (PTV) and V100(body) are the volumes of the $100 \%$ prescription dose within the PTV and body, respectively, and $V_{\mathrm{PTV}}$ is the volume of the PTV. For head-and-neck

TABLE I. Comparison of clinically delivered treatments with UIMAT treatment plans. Note that some patients had multiple target volumes with distinct dose

\begin{tabular}{|c|c|c|c|c|c|c|c|}
\hline & Patient & Site & Dose pres. (Gy) & Clinical & UIMAT & Clinical & UIMAT \\
\hline \multirow{3}{*}{ Head and neck } & 2 & Rt. parotid & 60 & $2 \times 225^{\circ}(116 \mathrm{CP})$ & $1 \times 225^{\circ}(55 \mathrm{CP})$ & 95 & 68 \\
\hline & 3 & Larynx/neck & $70 / 56$ & $2 \times 360^{\circ}(182 \mathrm{CP})$ & $1 \times 360^{\circ}(87 \mathrm{CP})$ & 151 & 171 \\
\hline & 4 & Parotids/neck & $70 / 56$ & $2 \times 360^{\circ}(182 \mathrm{CP})$ & $1 \times 360^{\circ}(89 \mathrm{CP})$ & 151 & 201 \\
\hline \multirow{4}{*}{ Lung } & 7 & Lt. lung & 60 & $2 \times 225^{\circ}(116 \mathrm{CP})$ & $1 \times 225^{\circ}(63 \mathrm{CP})$ & 94 & 67 \\
\hline & 8 & Rt. lung & 60 & 6 fields (21 CP) & $1 \times 192^{\circ}(61 \mathrm{CP})$ & 237 & 126 \\
\hline & 9 & Rt. lung & 60 & $2 \times 210^{\circ}(108 \mathrm{CP})$ & $1 \times 210^{\circ}(63 \mathrm{CP})$ & 90 & 98 \\
\hline & 10 & $\begin{array}{l}\text { Lt. lung and } \\
\text { mediastinum }\end{array}$ & 50 & $2 \times 360^{\circ}(181 \mathrm{CP})$ & $1 \times 360^{\circ}(93 \mathrm{CP})$ & 149 & 100 \\
\hline Prostate & 15 & Prostate & 66 & $2 \times 360^{\circ}(182 \mathrm{CP})$ & $1 \times 360^{\circ}(96 \mathrm{CP})$ & 151 & 139 \\
\hline
\end{tabular}
levels (e.g., Patient 3 had both a 70 Gy and a 56 Gy target volume). Approximate treatment delivery times are given.

Note: pres, prescription; Rt, right; Lt, left; UIMAT, unified intensity modulated arc therapy; CP, control points. 
cases, mean doses for parotids, oral cavity, and larynx, and maximum doses for cord, brainstem, and larynx were analyzed. For lung cases, mean doses for lung, esophagus, and heart were recorded as well as the lung $V 20$ and the maximum cord dose. For prostate cases, mean doses for rectum, bladder, bowel, and femurs were analyzed. The threshold for statistical significance was set at a $p$-value of 0.05 .

The deliverability of UIMAT plans was tested on a Varian TrueBeam linear accelerator (Varian Medical Systems, Palo Alto). The accuracy of dose calculations and delivery was verified by measurements with the ArcCheck Phantom (Sun Nuclear).

\section{RESULTS}

\section{A. Feasibility}

The UIMAT plans were generated for 15 cases, as summarized in Table I. UIMAT plans employed only one arc, while most of the clinical VMAT plans required two arcs. The number of MLC control points in the UIMAT plans was less than the VMAT plans for all but one case (see Table I), but more than in the IMRT plans. The estimated delivery times for UIMAT plans were not significantly different from the VMAT plans $(p=0.22)$ but they were significantly faster than multiple-field IMRT plans $(p=0.01)$. No significant difference in delivery time was observed between the UIMAT plans and the VMAT and IMRT plans taken together $(p=0.75)$.

An ArcCheck measurement in each treatment site was made to verify the accuracy of UIMAT dose calculation and feasibility of delivery. All tested plans had a gamma passing rate $^{16}$ of greater than $95 \%$ using our standard clinical parameters of 3\% dose difference and $3 \mathrm{~mm}$ distance-to-agreement.

\section{B. Plan comparison}

\section{B.1. Head and neck}

The dose metrics used in the evaluation of the headand-neck cases are presented in Table II. Plans which have multiple PTVs, as listed in Table I, have one CI reported for each PTV. For patient 5, the low doses associated with most organs at risk (OARs) are related to the small treatment volume which is restricted to the laryngeal region of the neck. For the five head-and-neck cases, the overall average of the mean OAR doses in Table II was reduced by $8.4 \%$ $(p<0.001)$ using UIMAT plans compared to the clinical VMAT or IMRT plans. As a specific example, comparison of the dose distributions and DVHs between a UIMAT and a VMAT plan for a head-and-neck case is shown in Fig. 3. It shows that the UIMAT plan produced lower OAR doses with similar PTV coverage compared with the clinical VMAT plan.

\section{B.2. Lung}

The dose metrics used in the evaluation of the lung cases are presented in Table III. UIMAT yielded significant reductions for the mean doses for heart and esophagus, and maximum cord dose compared with clinical IMRT or VMAT plans, while the difference in $V 20$, mean lung dose, conformity index, and mean PTV dose is not statistically significant. The overall average of the mean OAR doses in Table III was reduced by $5.7 \%(p<0.001)$ using UIMAT plans compared with clinical IMRT or VMAT plans.

As a specific example, comparison of the dose distributions and DVHs between a UIMAT and a five-field IMRT plan for a lung case is shown in Fig. 4. The UIMAT plan produced more conformal and uniform dose to the PTV and lower doses

TABLE II. Dose volume parameters of interest for five head-and-neck cases. The mean PTV dose is represented as a percentage of the prescription dose. Certain OARs were not contoured for some patients, either because the OAR was well outside the treatment volume or because it was completely enclosed within the PTV. The conformity indices correspond to the target volumes listed in the third-last column, and similarly for the mean PTV doses. Since not all patients had the same number of PTV dose levels, $P$ values and average doses for PTVs were calculated for the highest dose level only.

\begin{tabular}{|c|c|c|c|c|c|c|c|c|c|c|c|}
\hline No. & Plan & $\begin{array}{c}\text { Oral } \\
\text { cavity } \\
\bar{D}(\mathrm{~Gy})\end{array}$ & $\begin{array}{c}\text { Left } \\
\text { parotid } \\
\bar{D}(\mathrm{~Gy})\end{array}$ & $\begin{array}{l}\text { Right } \\
\text { parotid } \\
\bar{D}(\mathrm{~Gy})\end{array}$ & $\begin{array}{l}\text { Larynx } \\
\bar{D}(\mathrm{~Gy})\end{array}$ & $\begin{array}{c}\text { Larynx } \\
D_{\max } \\
(\mathrm{Gy})\end{array}$ & $\begin{array}{c}\text { Brainstem } \\
D_{\max } \\
(\mathrm{Gy})\end{array}$ & $\begin{array}{l}\text { Cord } \\
D_{\max } \\
(\mathrm{Gy})\end{array}$ & $\begin{array}{c}\text { PTV(s) } \\
\text { Pres. (Gy) }\end{array}$ & $\begin{array}{c}\text { Conformity } \\
\text { index }\end{array}$ & $\operatorname{PTV}(\mathrm{s}) \bar{D}(\%)$ \\
\hline \multirow{2}{*}{1} & Clinical & 28.4 & 6.9 & - & 31.8 & 62.3 & 15.0 & 22.4 & \multirow{2}{*}{$64 / 60 / 54$} & $0.21 / 0.77 / 0.63$ & $99.8 / 101.5 / 103.5$ \\
\hline & UIMAT & 22.6 & 2.4 & - & 26.8 & 61.2 & 9.5 & 24.5 & & $0.14 / 0.79 / 0.63$ & $101.9 / 102.6 / 103.7$ \\
\hline \multirow{2}{*}{2} & Clinical & 30.0 & 5.9 & 61.0 & 27.8 & 62.6 & 13.8 & 36.7 & \multirow{2}{*}{60} & 0.85 & 101.3 \\
\hline & UIMAT & 27.7 & 3.3 & 60.8 & 26.3 & 63.1 & 10.9 & 37.4 & & 0.80 & 101.5 \\
\hline \multirow{2}{*}{3} & Clinical & 33.2 & 25.9 & 25.6 & - & - & 32.2 & 36.9 & \multirow{2}{*}{$70 / 56$} & $0.83 / 0.79$ & $100.0 / 101.8$ \\
\hline & UIMAT & 29.9 & 23.1 & 22.4 & - & - & 33.3 & 44.3 & & $0.70 / 0.70$ & $101.7 / 102.6$ \\
\hline \multirow{2}{*}{4} & Clinical & 39.3 & 25.3 & 25.4 & 48.6 & 65.1 & 44.3 & 44.8 & \multirow{2}{*}{$70 / 56$} & $0.78 / 0.72$ & $100.6 / 104.5$ \\
\hline & UIMAT & 37.3 & 22.5 & 22.9 & 47.7 & 58.0 & 46.0 & 23.4 & & $0.82 / 0.71$ & $100.7 / 103.6$ \\
\hline \multirow{2}{*}{5} & Clinical & 0.2 & 0.2 & 0.2 & 55.5 & 64.2 & 0.2 & 0.2 & \multirow{2}{*}{$61 / 50$} & $0.89 / 0.73$ & $101.0 / 109.6$ \\
\hline & UIMAT & 0.2 & 0.2 & 0.2 & 55.5 & 64.0 & 39.8 & 23.6 & & $0.93 / 0.73$ & $101.6 / 108.6$ \\
\hline \multirow{2}{*}{ Avg. } & Clinical & 26.2 & 12.8 & 28.0 & 40.9 & 63.6 & 21.1 & 31.8 & & 0.72 & 102.4 \\
\hline & UIMAT & 23.6 & 10.3 & 26.6 & 39.0 & 61.6 & 20.0 & 34.9 & & 0.7 & 102.9 \\
\hline$P$ value & & 0.02 & 0.01 & 0.09 & 0.09 & 0.17 & 0.23 & 0.04 & & 0.11 & 0.08 \\
\hline
\end{tabular}

Note: PTV, planning target volume; OAR, organ at risk; UIMAT, unified intensity modulated arc therapy; $\bar{D}$, mean dose; $\mathrm{D}_{\text {max }}$, maximum dose; avg, average. 

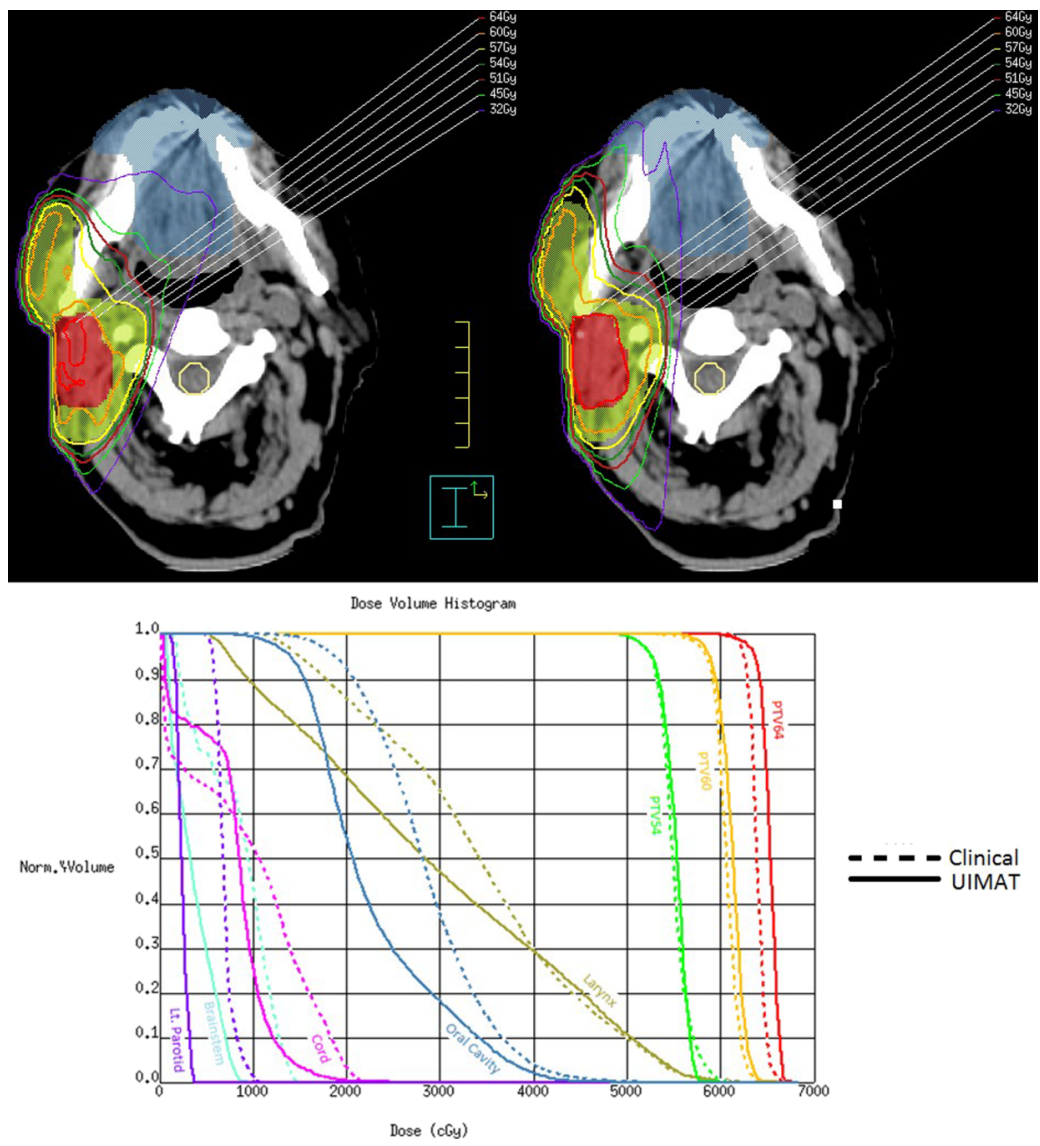

FIG. 3. Comparison of dose distributions between a clinical VMAT plan (top left) and a UIMAT plan (top right) and corresponding dose volume histograms (bottom) for a head-and-neck case (patient 1).

to left lung, esophagus, and spinal cord compared with the IMRT plan.

\section{B.3. Prostate}

The dosimetric parameters of UIMAT and clinical VMAT plans for five prostate cases are shown in Table IV. The multiple conformity indices for patient 14 correspond to the multiple PTVs within the plan. No significant dosimetric difference was observed between UIMAT and VMAT plans for the prostate cases studied. The overall average of the mean OAR doses listed in Table IV was reduced by $3.5 \%$ $(p=0.009)$ using UIMAT compared with VMAT plans. As a specific example, comparison of the dose distributions and

TABLE III. Dose volume parameters of interest for five lung cases. The mean PTV dose is represented as a percentage of the prescription dose.

\begin{tabular}{|c|c|c|c|c|c|c|c|c|}
\hline No. & Plan & $\begin{array}{c}\text { Lung } V 20 \\
(\%)\end{array}$ & $\begin{array}{c}\text { Lung } \bar{D} \\
\quad(\mathrm{~Gy})\end{array}$ & $\begin{array}{c}\text { Esophagus } \\
\bar{D} \text { (Gy) }\end{array}$ & $\begin{array}{l}\text { Heart } \bar{D} \\
\quad(\mathrm{~Gy})\end{array}$ & $\begin{array}{l}\text { Cord } D_{\max } \\
\quad(\mathrm{Gy})\end{array}$ & CI & PTV $\bar{D}(\%)$ \\
\hline \multirow{2}{*}{6} & Clinical & 15.1 & 9.8 & 17.1 & 1.2 & 29.5 & 0.72 & 101.7 \\
\hline & UIMAT & 14.9 & 9.2 & 15.7 & 1.0 & 27.9 & 0.87 & 102.0 \\
\hline \multirow{2}{*}{7} & Clinical & 21.9 & 14.0 & 22.4 & 10.1 & 35.6 & 0.87 & 101.3 \\
\hline & UIMAT & 22.1 & 14.0 & 21.2 & 8.3 & 34.8 & 0.91 & 101.3 \\
\hline \multirow{2}{*}{8} & Clinical & 30.5 & 17.2 & 25.0 & 11.0 & 47.8 & 0.79 & 103.5 \\
\hline & UIMAT & 27.8 & 15.5 & 22.7 & 10.6 & 47.4 & 0.91 & 101.5 \\
\hline \multirow{2}{*}{9} & Clinical & 20.7 & 11.8 & 24.4 & 4.8 & 43.3 & 0.78 & 101.3 \\
\hline & UIMAT & 20.7 & 11.5 & 23.3 & 3.8 & 41.2 & 0.81 & 101.3 \\
\hline \multirow{2}{*}{10} & Clinical & 35.6 & 18.6 & 36.2 & 30.6 & 43.4 & 0.92 & 100.2 \\
\hline & UIMAT & 34.4 & 18.1 & 35.5 & 29.4 & 42.5 & 0.88 & 101.0 \\
\hline \multirow{2}{*}{ Avg. } & Clinical & 24.8 & 14.3 & 25.0 & 11.5 & 39.9 & 0.82 & 101.6 \\
\hline & UIMAT & 24.0 & 13.7 & 23.7 & 10.6 & 38.8 & 0.88 & 101.4 \\
\hline$P$ value & & 0.12 & 0.05 & 0.003 & 0.01 & 0.01 & 0.08 & 0.37 \\
\hline
\end{tabular}

Note: PTV, planning target volume; CI, conformity index; V20, percent volume of lung receiving at least $20 \mathrm{~Gy} ; \bar{D}$, mean dose; $D_{\max }$, maximum dose; UIMAT, unified intensity modulated arc therapy; avg, average. 

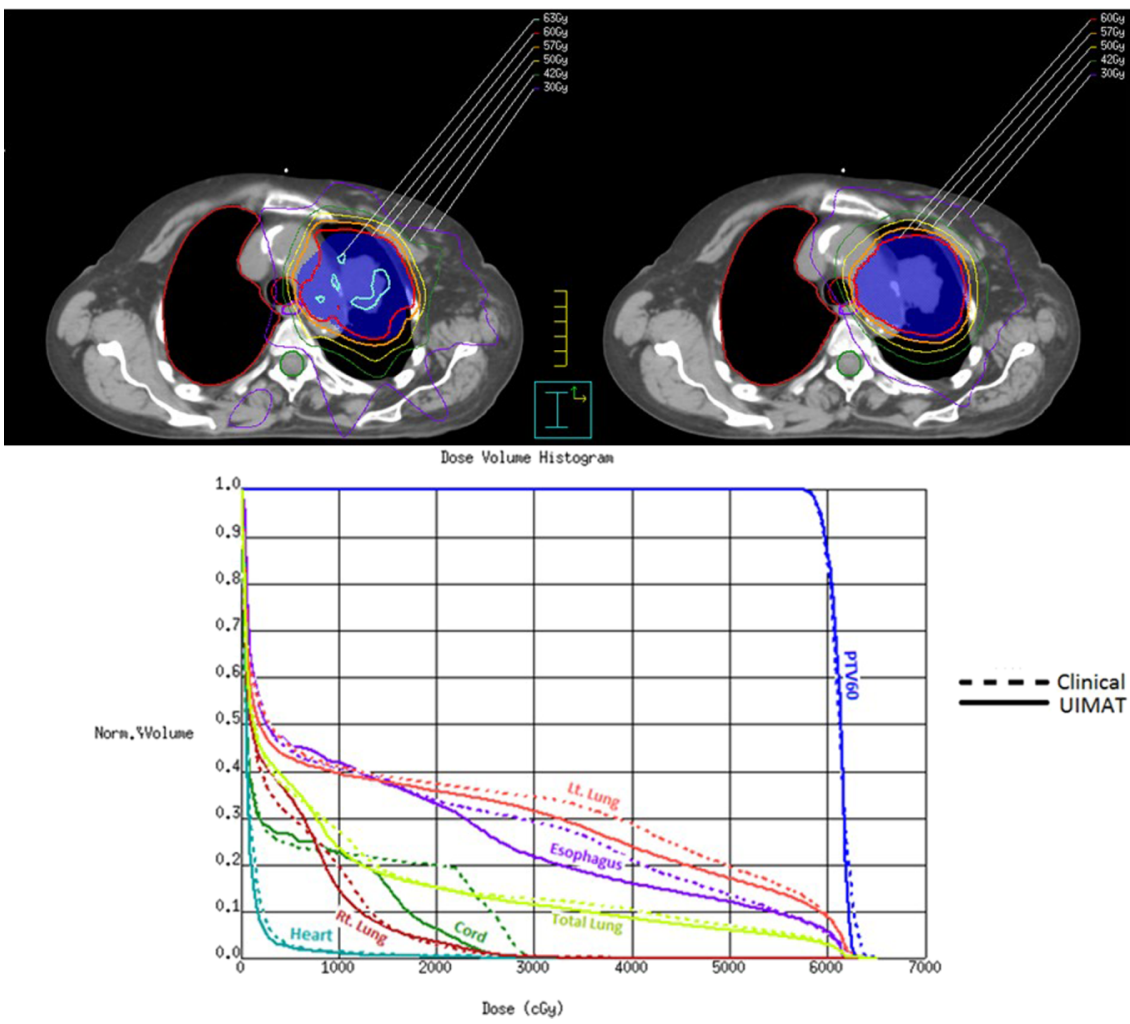

FIG. 4. Comparison of dose distributions between a clinical IMRT plan (top left) and a UIMAT plan (top right) and corresponding dose volume histograms (bottom) for a lung case (patient 6).

DVHs between a UIMAT and a VMAT plan for a prostate case is shown in Fig. 5. In this case, the UIMAT plan is dosimetrically similar to the clinical VMAT plan.

\section{DISCUSSION}

We have shown that it is feasible to optimize and deliver UIMAT which combines VMAT and IMRT within the same arc. We have also shown how the degree of intensity modulation can be naturally incorporated into an algorithm which dynamically varies the angular density of the beam control points. Compared with clinical VMAT or IMRT plans, UIMAT has the potential to produce efficient and superior radiation dose distributions, especially for complex anatomy such as in head-and-neck cancers. On the other hand, for the sites with more rotational symmetry, such as prostate, UIMAT may not yield significant advantages as it resulted

TABLE IV. Dose-volume parameters of interest for five prostate cases. The bowel was not contoured for two patients as it lay well outside the treatment volume. The conformity indices correspond to the target volumes listed in the third-last column, and similarly for the mean PTV doses. Since not all patients had the same number of PTV dose levels, $P$ values for PTVs were calculated for the highest dose level only.

\begin{tabular}{|c|c|c|c|c|c|c|c|c|c|}
\hline No. & Plan & $\begin{array}{l}\text { Rectum } \\
\bar{D}(\mathrm{~Gy})\end{array}$ & $\begin{array}{l}\text { Bladder } \\
\bar{D}(\mathrm{~Gy})\end{array}$ & $\begin{array}{l}\text { Bowel } \bar{D} \\
\quad(\mathrm{~Gy})\end{array}$ & $\begin{array}{l}\text { Lt. femur } \bar{D} \\
\text { (Gy) }\end{array}$ & $\begin{array}{l}\text { Rt. femur } \bar{D} \\
\text { (Gy) }\end{array}$ & $\begin{array}{c}\text { PTV(s) pres. } \\
(\mathrm{Gy})\end{array}$ & $\begin{array}{c}\text { Conformity } \\
\text { index }\end{array}$ & PTV $\bar{D}(\%)$ \\
\hline \multirow{2}{*}{11} & Clinical & 41.4 & 15.0 & - & 19.0 & 22.6 & \multirow{2}{*}{76} & 0.83 & 101.4 \\
\hline & UIMAT & 39.2 & 12.3 & - & 16.3 & 17.6 & & 0.85 & 101.9 \\
\hline \multirow{2}{*}{12} & Clinical & 41.4 & 54.3 & 53.1 & 22.4 & 16.5 & \multirow{2}{*}{66} & 0.82 & 98.2 \\
\hline & UIMAT & 42.8 & 55.5 & 52.2 & 20.8 & 20.8 & & 0.87 & 98.1 \\
\hline \multirow{2}{*}{13} & Clinical & 34.2 & 34.2 & 28.8 & 16.1 & 15.3 & \multirow{2}{*}{45} & 0.78 & 103.4 \\
\hline & UIMAT & 32.2 & 33.4 & 26.3 & 16.2 & 13.8 & & 0.84 & 101.9 \\
\hline \multirow{2}{*}{14} & Clinical & 45.6 & 45.5 & 31.6 & 22.9 & 21.5 & \multirow{2}{*}{$76 / 50.4$} & $0.91 / .0 .60$ & $102.0 / 107.0$ \\
\hline & UIMAT & 46.4 & 43.8 & 29.7 & 19.4 & 21.6 & & $0.88 / 0.59$ & $102.2 / 106.6$ \\
\hline \multirow{2}{*}{15} & Clinical & 32.3 & 17.9 & - & 23.4 & 23.3 & \multirow{5}{*}{66} & 0.91 & 100.9 \\
\hline & UIMAT & 32.1 & 17.8 & - & 21.7 & 22.5 & & 0.84 & 100.9 \\
\hline \multirow{2}{*}{ Average } & Clinical & 39.0 & 33.4 & 37.85 & 20.8 & 19.8 & & 0.81 & 102.1 \\
\hline & UIMAT & 38.6 & 32.6 & 36.1 & 18.9 & 19.3 & & 0.81 & 101.9 \\
\hline$P$ value & & 0.29 & 0.16 & 0.03 & 0.02 & 0.37 & & 0.39 & 0.26 \\
\hline
\end{tabular}

Note: PTV, planning target volume; Lt, left; Rt, right; pres, prescription; $\bar{D}$, mean dose; UIMAT, unified intensity modulated arc therapy. 

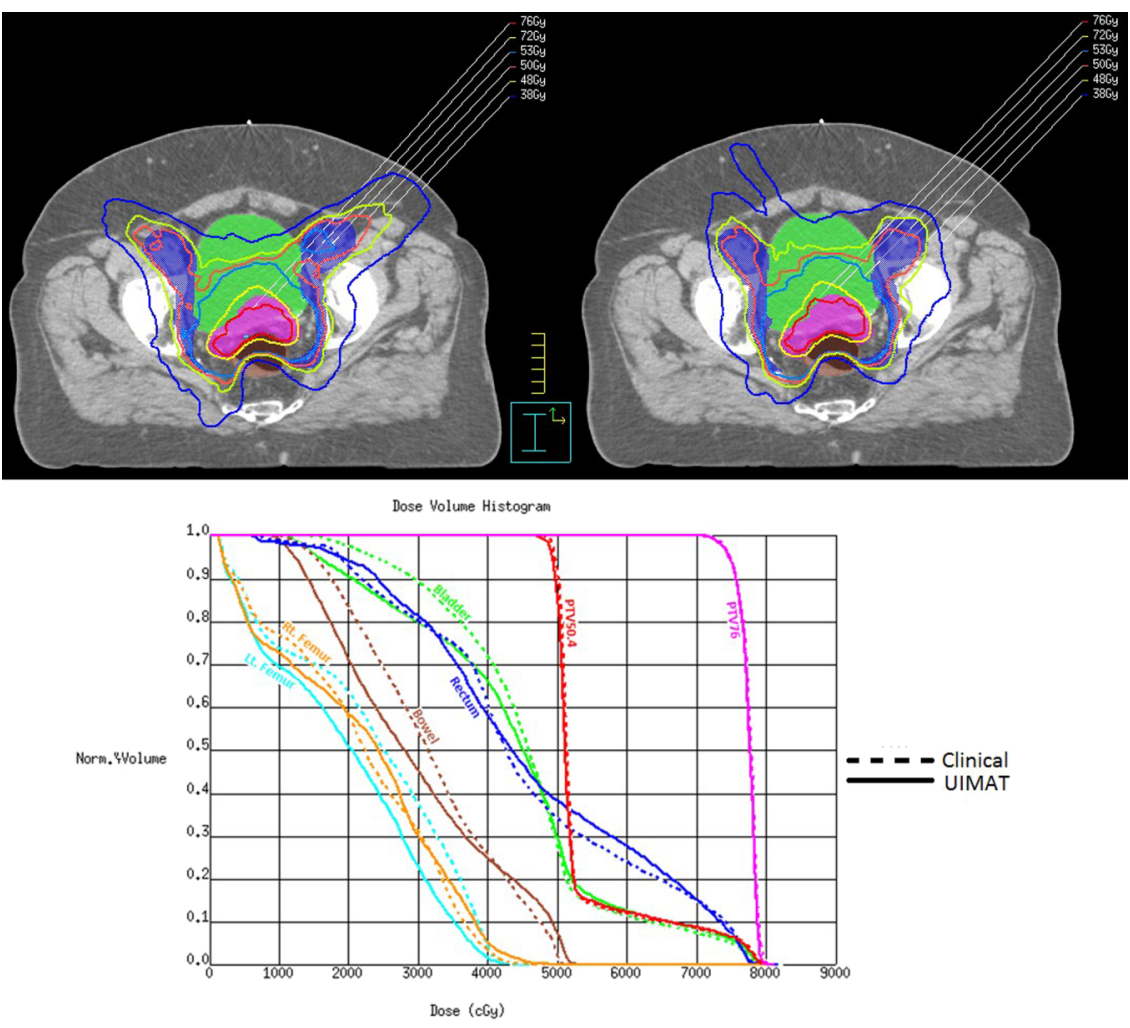

FIG. 5. Comparison of dose distributions between a clinical VMAT plan (top left) and a UIMAT plan (top right) and corresponding dose volume histograms (bottom) for a prostate case (patient 14).

in plans with comparable dosimetric performance but less efficient delivery compared with VMAT.

One of the virtues of our proposal is that the switch from the current VMAT technique to our unified approach would, in principle, be seamless. Treatment planning for UIMAT would be the same as for VMAT as no selection of static beam directions is required. Similarly, radiation therapists delivering the treatment would not need to perform any additional steps beyond what is commonly done for stand-alone VMAT delivery, although they must be made aware of the stopped gantry during IMRT phases of UIMAT delivery. Another benefit of our proposal is the ease at which it can be incorporated into a working clinical system, thus decreasing both cost and time between conception and clinical implementation. Of course, in practice, the introduction of this technique, as with all new techniques, would require additional quality assurance and testing.

Compared with other IMRT/VMAT combination techniques, ${ }^{7-12}$ our proposal offers simplicity in integration, optimization, and delivery. Our UIMAT proposal is not simply an IMRT/VMAT hybrid technique. It would be more correct to refer to it as a more fully realized implementation of VMAT with fuller range of gantry speed that even permits gantry stalls, thus allowing for optimal beam modulation. What we have demonstrated is one specific implementation of UIMAT, and in principle, it could be generalized further. UIMAT could be further developed with more robust segmentation and optimization algorithms, as well as more degrees of freedom such as collimator angle, couch position, and couch angle.
Such future developments could hopefully address certain limitations within our initial implementation of UIMAT. For example, once the initial gantry directions are chosen for IMRT phases at the start of optimization, they cannot be altered at a later point in the optimization. For most cases, this should not be a problem, except if the objective function changes dramatically between initial and final optimization. It should be noted that the same is true for VMAT and IMRT planning. After the fluence map conversion takes place, it becomes much easier for the optimization routine to become trapped in local minima.

In the planning comparison portion of this work, we acknowledge the usual biases and confounders inherent in such an approach. For example, there is a natural tendency toward demonstrating that a new treatment is superior to the standard-of-care. More effort may be spent optimizing the new technique or choosing comparison endpoints that naturally favor the new method. Unfortunately, requiring the matching of equal planning effort is not practical, but we were conscious in avoiding "overoptimizing" the UIMAT plans. In this retrospective study, the actual difference in planning time was not logged precisely. Depending on the complexity of the case, clinical planning in our experience requires 1-4 $\mathrm{h}$. To ensure a fair planning comparison, we restricted UIMAT planning times to fall within a similar range.

We acknowledge that the number of cases tested in this feasibility study is very limited and so the statistics reported only highlight general trends. More cases are needed for each treatment site to confirm the conclusions reached. Finally, as 
mentioned earlier, dynamic jaws are needed when treating large volumes with a single arc due to the finite speed and length of the MLC leaves. This is an obvious advantage for UIMAT as it allows more freedom for collimating the beam; however comparable degrees of freedom were still available in our clinical plans but required more than one arc with differing jaw positions.

Compared to other published techniques ${ }^{7-12}$ combining IMRT and VMAT, UIMAT is unique in that it possesses all of the following features: (1) it creates VMAT and IMRT phases automatically; (2) it optimizes VMAT and IMRT phases simultaneously; (3) the VMAT and IMRT phases are combined and delivered in a single dynamic arc; (4) the algorithm has been implemented on a commercial treatment planning system; (5) the UIMAT plans have been validated using the ArcCheck phantom, delivered in clinical mode on a Varian TrueBeam linear accelerator. Theoretically, it is known that the increased degrees of freedom afforded by the IMRT phases of the arc will, in principle, lead to a superior plan, all other things being equal. What we have shown is that this appears to be the case, even with this simple initial implementation. Further improvements in the optimization beyond what is possible within a commercial treatment planning software should lead to even better results. Last but not least, our work shows that the UIMAT delivery speed is improved over IMRT or when multiple VMAT arcs are required.

\section{CONCLUSIONS}

We have demonstrated the feasibility of a novel radiation therapy delivery technique termed UIMAT. This technique combines VMAT and IMRT optimization concurrently and delivers radiation in a single arc. The optimal fixedgantry IMRT phases are chosen automatically during the optimization. Optimization of both the VMAT and fixedgantry IMRT phases of delivery occurs simultaneously, and the final plan is an integrated UIMAT plan. Initial results show that the UIMAT has the potential to be superior to either stand-alone IMRT or VMAT in terms of dose distribution quality and efficiency of delivery.

\section{ACKNOWLEDGMENT}

The authors would like to thank the London Regional Cancer Program for funding this work through a Catalyst Grant.

a) Author to whom correspondence should be addressed. Electronic mail: douglas.hoover@lhsc.on.ca

${ }^{1} \mathrm{C}$. X. Yu, "Intensity-modulated arc therapy with dynamic multileaf collimation: An alternative to tomotherapy," Phys. Med. Biol. 40(9), 1435-1499 (1995).

${ }^{2}$ K. Otto, "Volumetric modulated arc therapy: IMRT in a single gantry arc," Med. Phys. 35(1), 310-317 (2008).

${ }^{3}$ D. M. Shepard et al., "An arc-sequencing algorithm for intensity modulated arc therapy," Med. Phys. 34(2), 464-470 (2007).

${ }^{4} \mathrm{X}$. Jiang et al., "Planning analysis for locally advanced lung cancer: Dosimetric and efficiency comparisons between intensity-modulated radiotherapy (IMRT), single-arc/partial-arc volumetric modulated arc therapy (SA/PA-VMAT)," Radiat. Oncol. 6, 140 (2011).

${ }^{5}$ D. Craft et al., "Multicriteria VMAT optimization," Med. Phys. 39(2), 686696 (2012).

${ }^{6} \mathrm{~F}$. Peng et al., "A new column-generation-based algorithm for VMAT treatment plan optimization," Phys. Med. Biol. 57(14), 4569-4588 (2012).

${ }^{7} \mathrm{R}$. Li and L. Xing, "Bridging the gap between IMRT and VMAT: Dense angularly sampled and sparse intensity modulated radiation therapy," Med. Phys. 38(9), 4912-4919 (2011).

${ }^{8}$ H. Kim et al., "Dose optimization with first-order total-variation minimization for dense angularly sampled and sparse intensity modulated radiation therapy (DASSIM-RT)," Med. Phys. 39(7), 4316-4327 (2012).

${ }^{9} \mathrm{C}$. Wang et al., "Arc-modulated radiation therapy (AMRT): A single-arc form of intensity-modulated arc therapy," Phys. Med. Biol. 53(22), 62916303 (2008)

${ }^{10}$ M. M. Matuszak et al., "FusionArc optimization: A hybrid volumetric modulated arc therapy (VMAT) and intensity modulated radiation therapy (IMRT) planning strategy," Med. Phys. 40(7), 071713 (10pp.) (2013).

${ }^{11}$ T. Gevaert et al., "Implementation of HybridArc treatment technique in preoperative radiotherapy of rectal cancer: Dose patterns in target lesions and organs at risk as compared to helical tomotherapy and RapidArc," Radiat. Oncol. 7, 120 (2012).

${ }^{12} \mathrm{~J}$. L. Robar and C. Thomas, "HybridArc: A novel radiation therapy technique combining optimized dynamic arcs and intensity modulation," Med. Dosim. 37(4), 358-368 (2012).

${ }^{13} \mathrm{~K}$. Bzdusek et al., "Development and evaluation of an efficient approach to volumetric arc therapy planning," Med. Phys. 36(6), 2328-2339 (2009).

${ }^{14}$ L. B. Marks et al., "Use of normal tissue complication probability models in the clinic," Int. J. Radiat. Oncol., Biol., Phys. 76(3), S10-S19 (2010).

${ }^{15}$ R. Oozeer et al., "Dosimetric evaluation of conformal radiotherapy: Conformity factor," Cancer Radiother. 4(3), 207-216 (2000).

${ }^{16} \mathrm{D}$. A. Low et al., "A technique for the quantitative evaluation of dose distributions," Med. Phys. 25(5), 656-661 (1998). 